4. Colditz GA, Hankinson SE, Hunter DJ, et al. The use of oestrogens and progestins and the risk of breast cancer in postmenopausal women. N Engl J Med 1995; 332 : 1589-93.

5. Collaborative Group on Hormonal factors in Breast Cancer. Breast cancer and hormone replacement therapy: collaborative reanalysis of data from 51 epidemiological studies of
52,705 women with breast cancer and 108,411 women without breast cancer. Lancet 1997; 350 :

1047-1059.

6. Shairer C, Gail M, Byrne C, et al. Estrogen replacement therapy and breast cancer survival in a large screening study. J Natl Cancer Inst 1999; 91: 264-70.

7. Bonnier P, Sakr R, Bennessay $F$, et al. Effects of hormone replacement therapy for menopause on prognostic factors of breast cancer. Gynecol Obstet Fertil 2000; 28: 745-53.

8. Santen RJ, Pinkerton J, McCartney C, Petroni GR. Risk of breast cancer with progestins in combination with estrogen as hormone replacement therapy. J Clin Endocrinol Metab 2001; 86: 16-23.

9. Hulley S, Furberg C, BarrettConnor $\varepsilon$, et al. for the HERS Research

Group. Noncardiovascular disease outcomes

during 6.8 years of hormone

therapy. Heart and

estrogen/progestin

replacement study followup (HERS II). JAMA 2002; 288: 58-66.

10. Expertise Collective Inserm. Hormone replacement therapy. Influence on cardiovascular risk. Paris: Éditions Inserm, 2000.

\title{
NOUVELLE
}

\section{Mise en évidence de processus de duplications en bloc dans le génome des vertébrés}

Anne Miquelis, Laurent Abi-Rached, André Gilles, Pierre Pontarotti

> L'hypothèse selon laquelle le génome des vertébrés aurait évolué via plusieurs tours de polyploïdisations a déjà plus de trente ans [1]. Le génome des actuels vertébrés à mâchoires aurait dû conserver la trace de ces phénomènes sous la forme d'un réseau de régions paralogues (voir glossaire) au sein desquelles toutes les paires de gènes dupliqués auraient subi cet événement simultanément. L'analyse de la carte du génome humain [2] a permis d'identifier différentes régions de paralogie de ce type parmi lesquelles celle qui contient les gènes codant pour les éléments du complexe majeur d'histocompatibilité (CMH) [3] pour lequel le processus de duplication s'est vraisemblablement produit il y a au moins 420 millions d'années [4]. Des gènes ancres à évolution plus lente ont été mis en évidence à proximité des gènes humains du CMH [4]. Afin de savoir si le génome de notre ancêtre a subi des duplications en bloc, nous avons choisi [5]: (1) d'analyser en détail les gènes dupliqués présents dans la série de paralogie du CMH (analyses phylogénétiques et cartes), (2) de vérifier I'hypothèse selon laquelle la duplication en bloc serait survenue après la radiation des vertébrés et (3) de cloner les gènes équivalents chez l'amphioxus (ou lancelet), choisi comme animal modèle en raison de sa position au sein de l'arbre évolutif des métazoaires (Figure 1).

Parmi les 18 gènes ancres définis comme tels par une recherche dans les bases de données de nouveaux gènes paralogues et de leurs relations phylogénétiques, onze ont été sélectionnés pour cette étude. Un cosmide (voir glossaire) cloné a ensuite été entièrement séquencé et analysé pour chaque gène ancre afin d'étudier le contenu et les relations phylogénétiques des gènes de l'amphioxus et de leurs correspondants humains (Figure 2). Les gènes avoisinant chaque gène ancre sur les cosmides correspondants ont alors été prédits par analyse bio-informatique. Un total de 41 gènes sont présents sur l'ensemble des cosmides séquencés (incluant les gènes ancres et les gènes avoisinants) (400 kilobases). Ces 41 gènes sont alors utilisés pour rechercher des séquences semblables dans les bases de données. Des similitudes significatives sont observées pour 34 gènes ( 7 gènes ne montrent pas de similitudes significatives avec les séquences présentes dans les bases de données), parmi lesquels 33 incluent des séquences humaines. L'analyse des jeux de données permet l'attribution du critère d'orthologie à 31 gènes de l'amphioxus parmi lesquels 9 sont des gènes ancres et 22 des gènes qui leur sont adjacents. L'analyse des relations phylogénétiques des gènes ancres de l'amphioxus permet de conclure que les duplications ayant engendré les différentes familles de gènes ancres chez les vertébrés se sont produites entre 766-420 millions d'années (pour la diversification des vertébrés osseux) et probablement entre 766528 millions d'années (pour la séparation des vertébrés à mâchoires).

L'analyse de la signification statistique de la distribution des gènes humains orthologues des gènes de l'amphioxus a été réalisée dans quatre régions de paralogie du CMH humain, sur les chromosomes 1, 6, 9 et 19 (Figure 2). Vingt-deux gènes de l'amphioxus situés au voisinage des gènes ancres clonés ont au moins un orthologue humain pour un total de 42 orthologues 
humains parmi lesquels seize sont localisés dans une région de paralogie du CMH. La distribution des gènes humains orthologues des gènes voisins des gènes ancres de l'amphioxus est statistiquement différente d'une distribution au hasard pour ces quatre régions ce qui indique l'existence d'un lien évolutif entre les régions génomiques de l'amphioxus et les quatre régions génomiques humaines. Le modèle le plus probable permettant d'expliquer le lien entre les régions génomiques de l'amphioxus et les régions de paralogie dans le CMH humain est l'existence d'un ancêtre génomique commun aux quatre régions génomiques humaines ayant subi une duplication en bloc après la séparation céphalochordés-vertébrés. Les régions de paralogie du CMH sont donc des régions parasynténiques (voir glossaire) qui ont été dupliquées en bloc au cours d'une période comprise entre 766 et 528 millions d'années.

L'aspect le plus frappant de l'évolution des régions dupliquées est le nombre de gènes fixés. La région génomique du chromosome 9 contient en effet deux fois le nombre de la plupart des gènes dérivés de la région génomique ancestrale contrairement aux trois autres régions. Ainsi, en termes d'organisation génomique, la région du chromosome 9 est beaucoup plus semblable à la région génomique ancestrale supposée que ne le sont les trois autres régions de paralogie du CMH. Le caractère ancestral de la région du chromosome 9 s'observe également au niveau des gènes. L'analyse du modèle de substitution (calcul du taux de changement des acides aminés le long de la séquence permettant d'estimer la vitesse d'évolution d'une protéine) des familles géniques ayant plus de deux paralogues dans les régions de paralogie du CMH (six familles paralogues) montre que les gènes de la région du chromosome 9 ont toujours un taux de substitution faible comparé aux trois autres régions paralogues ( 6 gènes sur 6 , ce qui est statistiquement significatif) induisant une évolution moins rapide des gènes de cette région. L'essence d'un tel phénomène reste obscure, mais divers processus peuvent être impliqués comme les contraintes fonctionnelles, les contraintes d'expression et de régulation (expression ubiquitaire versus spécifique d'un tissu).

Ce travail suggère que les régions dupliquées ont des destins différents: une région peut être «préservée » (contrainte fonctionnelle) et entretenir une fonction ancestrale alors que d'autres peuvent être plus «libres», conduisant potentiellement à l'émergence de nouveaux gènes et/ou familles géniques ce qui est supposé pour la « véritable » région génomique du CMH sur le chromosome 6 [4]. L'échantillonnage d'autres régions génomiques est nécessaire afin de déterminer à quel point ce phénomène peut être généralisé.

Cette étude valide donc l'hypothèse de la duplication en bloc des régions de paralogie du CMH et constitue la première étape démontrant que des processus de poly- ploïdisation se sont produits dans notre génome ancestral après la séparation céphalochordés/vertébrés et avant la radiation des gnathostomes, c'est-à-dire entre 766 et 528 millions d'années. En reconstruisant la région ancestrale des quatre régions de paralogie du $\mathrm{CMH}$, nous avons découvert un aspect inattendu de l'évolution des régions dupliquées: une région a conservé un état ancestral à la fois en terme d'organisation et de profil de substitution des gènes. Notre génome comporte donc une grande région apparemment sous sélection négative (processus qui freine l'évolution des séquences; par exemple, une contrainte fonctionnelle) un concept appliqué peu fréquemment aux gènes pris individuellement ou aux segments de gènes. Il est important de déterminer dans quelle mesure cette découverte peut être généralisée à d'autres régions du

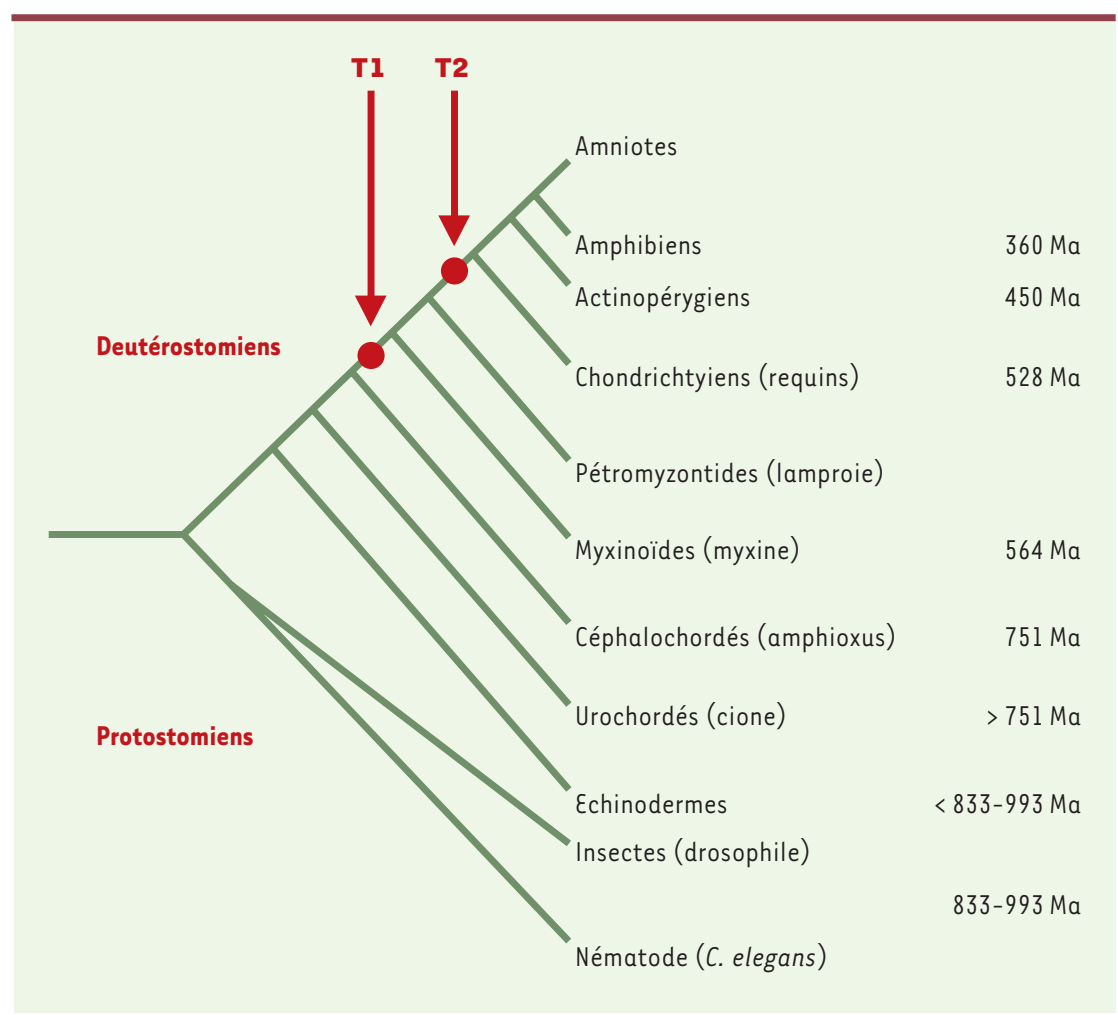

Figure 1. Cladogramme montrant les relations phylogénétiques des principaux groupes actuels (les longueurs de branches ne sont pas proportionnelles au temps de divergence séparant deux lignées terminales de leur ancêtre commun). Les temps de divergence sont calculés à partir des données moléculaires (sous l'hypothèse de l'horloge moléculaire) et calibrés à partir de données fossiles) et doivent être interprétés comme une échelle relative. $\mathrm{Tl}$ et $\mathrm{T} 2$ représentent le premier et le deuxième tour de duplication à grande échelle. Ma: millions d'années. 
génome. Ces analyses soulignent l'importance de la phylogénie et de la phylogénomie, non seulement pour révéler l'histoire évolutive de notre génome mais également pour une meilleure compréhension des organisations génomiques actuelles. $\diamond$ Demonstration of vertebrate genome bloc duplications

\section{REMERCIEMENTS}

Nous tenons à remercier Etienne Danchin et

Alexandre Vienne pour la relecture et les cri-

tiques apportées à ce manuscrit.

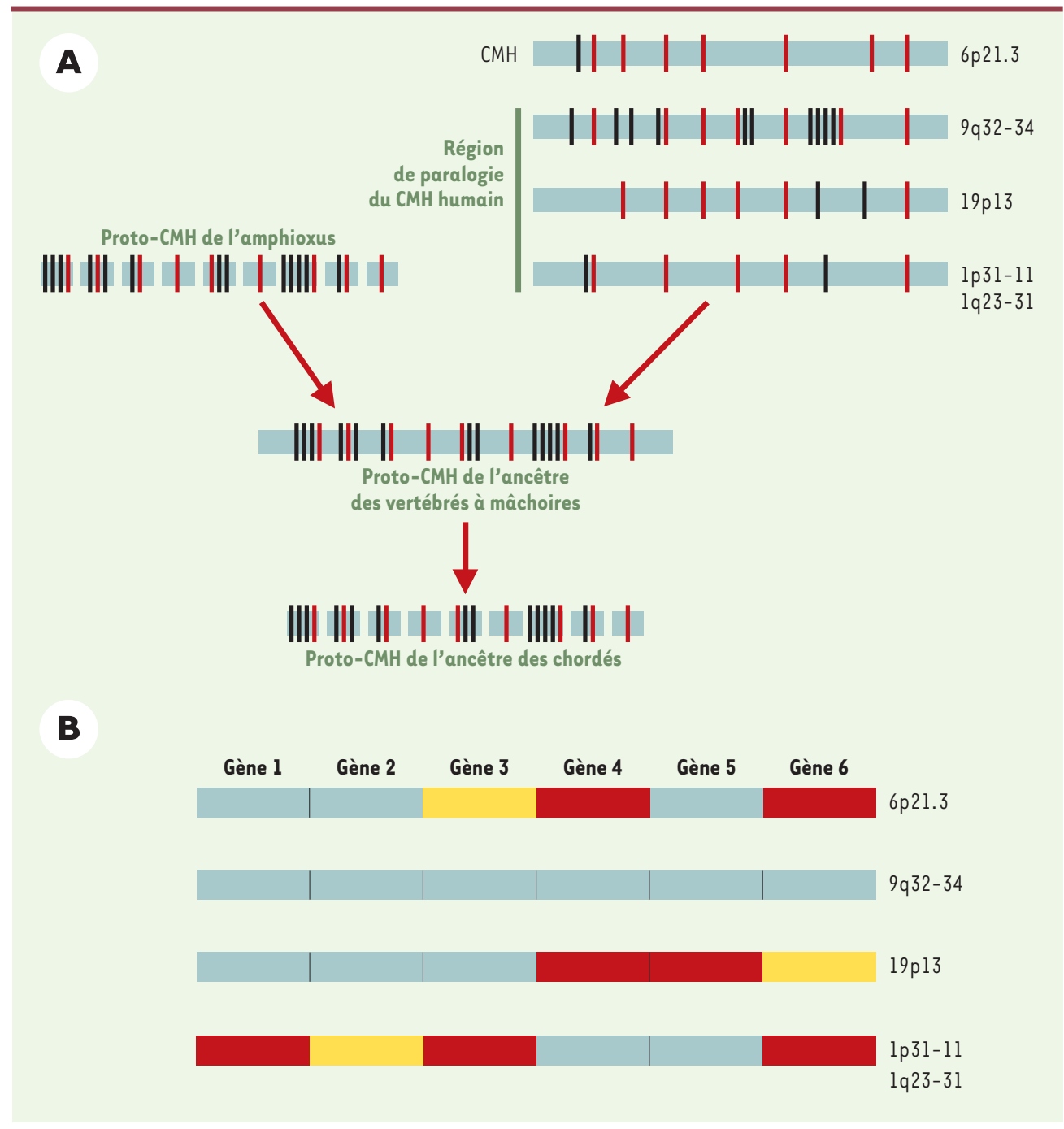

Figure 2. Évolution de la région du proto-CMH des chordés et de ses gènes. A. Hypothèse de reconstitution de l'évolution des régions génomiques paralogues du CMH. En comparant les régions de l'amphioxus et les régions de paralogie humaines, il est possible de reconstituer le proto-CMH ancestral des vertébrés à mâchoires. L'état de la région génomique du chordé ancestral reste inconnu en raison du caractère non lié des gènes de l'amphioxus. Les gènes ancres sont indiqués en rouge, les gènes adjacents aux gènes ancres de l'amphioxus et de leurs orthologues humains sont indiqués en noir. $B$. Profils de substitution au sein des gènes paralogues du CMH humain. Nous avons utilisé uniquement les gènes qui ont au moins trois copies dans les régions de paralogie du CMH. Pour chacun de ces gènes, nous avons comparé le taux de substitution des différents gènes paralogues. Les gènes indiqués en bleu ont un profil de substitution significativement plus lent que les gènes indiqués en rouge. Les boîtes jaunes correspondent aux paralogues perdus. La position relative des gènes dans les régions de paralogie du CMH humain est arbitraire.

\section{RÉFÉRENCES}

1. Ohno S. Evolution by Gene Duplication. Berlin: Springer, 1970.

2. Lundin LG. Evolution of the vertebrate genome as reflected in paralogous chromosomal regions in man and the house mouse. Genomics 1993; 16: 1-19.

3. Kasahara M, Nakaya J, Satta Y, Takahata N. Chromosomal duplication and the emergence of the adaptive immune system. Trends Genet 1997; 13: 90-2.

4. Abi-Rached L, McDermott MF, Pontarotti P. The MHC big bang. Immunol Rev 1999; 167: 33-44.

5. Abi-Rached L, Gilles A, Shiina T, Pontarotti P, Inoko H. Evidence of en bloc duplication in vertebrate genomes. Nat Genet 2002; 31: 100-5. 


\section{Paralogue}

Deux gènes sont dits «paralogues » s'ils sont issus d'un événement de duplication au sein du génome d'un même individu.

\section{Orthologue}

Gènes d'espèces différentes dont les séquences sont homologues, dérivent d'un même gène ancestral et ont divergé à la suite d'un événement de spéciation. Ils peuvent ou non avoir la même fonction

\section{Cosmides}

Fragments d'ADN insérés dans des plasmides et ayant des tailles comparables (de 30 à $50 \mathrm{kpb}$ ). Ce sont des vecteurs qui sont utilisés pour la construction de banques d'ADN génomique.

\section{Régions parasynténiques}

Terme qui fait référence à des régions génomiques dont la divergence est due à des événements de duplication.

NOUVELle

\title{
Maladies génétiques et lamines de type A: apport de la biologie structurale
}

\author{
Sophie Zinn-Justin
}

> L'enveloppe nucléaire sépare le nucléoplasme du cytoplasme et organise la structure du noyau dans toutes les cellules eucaryotes. Elle est formée de deux membranes concentriques percées de place en place par des pores nucléaires, qui permettent à certaines molécules d'être activement transportées depuis et vers le cytoplasme. La membrane nucléaire externe est en continuité physique avec le réticulum endoplasmique dont elle partage les fonctions. La membrane nucléaire interne est, quant à elle, associée à un réseau de protéines, la lamina nucléaire, qui est interposé entre la membrane et la chromatine [1]. Cette lamina nucléaire est essentiellement composée des lamines, protéines formant des filaments intermédiaires, et elle interagit avec un certain nombre de protéines ancrées à la membrane nucléaire interne (émerine, LAP2, LBR).

La lamina nucléaire fait aujourd'hui l'objet de nombreuses recherches. Quels sont ses partenaires dans le noyau? Quels rôles jouent l'ensemble de ces protéines dans la résistance mécanique de l'enveloppe nucléaire, l'organisation du noyau et la régulation du cycle cellulaire? Le rôle de la lamina varie-t-il selon le type cellulaire? L'intérêt des chercheurs a été aiguisé par la découverte récente d'un lien entre des mutations de certaines protéines de l'enveloppe nucléaire et des maladies héréditaires. En particulier, il a été montré que la dystrophie musculaire d'Emery-Dreifuss (EDMD) est due à des mutations soit du gène codant pour l'émerine [2], soit du gène codant pour des lamines de type A (lamines $A$ et $C$ ) [3]. Le gène codant pour des lamines de type $A$ est également muté dans cinq autres pathologies: (1) deux pathologies de type musculaire, la dystrophie musculaire des ceintures de type IB et la cardiomyopathie dilatée [3]; (2) une pathologie du tissu adipeux, la lipodystrophie de type Dunningan [3]; (3) une pathologie affectant le développement osseux, la dysplasie mandibuloacrale [4]; et (4) une pathologie du nerf périphérique, la maladie de Charcot Marie Tooth 2 [5].

Afin de mieux comprendre l'impact des mutations qui provoquent des pathologies héréditaires sur la structure et la fonction des protéines de l'enveloppe nucléaire, notre

\section{Cladogramme}

Arbre traduisant les relations de parenté entre des êtres vivants, établi à partir de la méthode cladistique (basée sur la notion d'homologie). Cet arbre exprime ainsi une hypothèse sur les parentés phylogénétiques entre plusieurs taxons (ensemble des organismes reconnus et définis dans chacune des catégories de la classification biologique hiérarchisée).
Laboratoire de structure des protéines, DIEP/DSV, bâtiment 152, CEA Saclay 91191 Gif-sur-Yvette Cedex, France. szinn@cea.fr

groupe s'intéresse à la caractérisation structurale de ces protéines par les approches suivantes: (1) détermination de la structure en solution des protéines dans leurs formes natives et mutées; (2) évaluation de l'impact des mutations sur la stabilité de ces protéines; (3) cartographie des surfaces d'interaction des protéines avec leurs partenaires biologiques; (4) positionnement des mutations par rapport à ces surfaces fonctionnellement importantes; et (5) identification de la structure tridimensionnelle des complexes protéiques auxquels participent des protéines de l'enveloppe nucléaire. Ce travail est mené en étroite collaboration avec des généticiens impliqués dans l'identification des mutations provoquant les différentes pathologies (équipe de Gisèle Bonne, Institut de Myologie, Hôpital PitiéSalpêtrière, Paris) et des biologistes cellulaires qui observent l'impact de ces mutations sur l'organisation et le fonctionnement des noyaux des cellules (équipes de JeanClaude Courvalin, Institut Jacques Monod, Paris, France, et d'Howard J. Worman, Columbia University, New York, USA) [6-8]. Récemment, nous avons publié la structure 